\title{
THE MASS DIFFERENCES OF THE $\Sigma$ HYPERONS
}

\author{
H. G. Dosch, R. Engelmann, H. Filthuth* , V. Hepp, E. Kluge, K. Marish**, \\ A. Minguzzi-Ranzi $* * *$ \\ University of Heidelberg, FRG, and CERN, Switzerland \\ (Presented by H. FILTHUTH)
}

\section{THE $\boldsymbol{\Sigma}^{-}-\boldsymbol{\Sigma}^{+}$MASS DIFFERENCE}

A selected sample of about 1,000 events of the types $K^{-}$(at rest) $p \rightarrow \Sigma-\pi^{+}$and $K^{-}$ (at rest ) $p \rightarrow \Sigma^{+} \pi^{-}$was used to determine the masses of the $\Sigma \pm$ hyperons from the measurement of the momenta of the pions, which are produced together with the $\Sigma$ hyperons. In order to deduce measurement errors the following conditions were required:

a) the visible track length of the pion is greater than $10 \mathrm{~cm}$;

b) the track length of the $\Sigma$ hyperon is greater than $0.2 \mathrm{~cm}$. $60^{\circ}$;

c) the dip-angle of the pion is smaller than

d) the angle between $K^{-}$meson and pion greater than $30^{\circ}$ and smaller than $150^{\circ}$.

Furthermore if only a fit assuming $\mathrm{K}^{-}$ interaction at rest was successful, the event was included in the sample. Since the magnitude of the measured pion momentum can be influenced by systematical errors in the magnetic field of the chamber, no attempt was made to give absolute mass values. However, the influence of systematic field and hydrogen density errors on the mass difference is small, compared to the statistical error of the sample. For the $\Sigma^{-}-\Sigma^{+}$mass difference, we obtain:

$$
M_{\Sigma^{-}-\Sigma^{+}}=(8.3 \pm 0.25) \mathrm{MeV} \text {. }
$$

\section{THE $\boldsymbol{\Sigma}^{-}-\boldsymbol{\Sigma}^{0}$ MASS DIFFERENCE}

For a selected sample of 37 events of the type: $\Sigma^{-} p \rightarrow \Sigma^{0} n, \Sigma^{0} \rightarrow \Lambda e^{+} e^{-}$with a visible $\Lambda$, the momentum of the $\Sigma^{0}$ has been determined from the vector sum of the measured momen-

\footnotetext{
* At University of Heidelberg, FRG and at CERN, Geneva, Switzerland.

** At Joint Institute for Nuclear Research, Dubna, USSR.

*** At University of Bologna, Italy.
}

ta of the electron and pusitron and the fitted momentum of the $\Lambda$ hyperon. The momentum of the $\Lambda$ was obtained from a $\Lambda$ decay fit, taking the $\Sigma^{-}$end point as the origin for the $\Lambda$. In all events the track length of the $\Sigma$ - hyperon was larger than $0.95 \mathrm{~cm}$ and smaller than $1.15 \mathrm{~cm}$; having a mean value of $L_{\Sigma}=1.05 \pm$ $\pm 0.01 \mathrm{~cm}$. The average momentum of the 37 $\Sigma^{0}$ 's is $(61.4 \pm 1.0) \mathrm{MeV} / \mathrm{c}$. The mass difference is quadratically related to this momentum by the expression:

$$
M_{\Sigma^{-}}-M_{\Sigma^{+}}=\left(1.29+0.95 \times 10^{-3}\right) P_{\Sigma^{0}}^{2} \mathrm{MeV} .
$$

We obtain

$$
M_{\Sigma^{-}}-M_{\Sigma^{0}}=(4.87 \pm 0.12) \mathrm{MeV}
$$

\section{SUMMAR Y}

$$
\begin{aligned}
& M_{\Sigma^{-}}-M_{\Sigma^{+}}=(8.30 \pm 0.25) \mathrm{MeV} ; \\
& M_{\Sigma^{-}-}-M_{\Sigma^{0}}=(4.87 \pm 0.12) \mathrm{MeV} ; \\
& M_{\Sigma^{0}}-M_{\Sigma^{+}}=(3.43 \pm 0.28) \mathrm{MeV} .
\end{aligned}
$$

Using $M_{\Sigma^{+}}=1189.4 \pm 0.2 \mathrm{MeV}$ from Rosenfeld et al. [1] we obtain

$$
\begin{aligned}
& M_{\Sigma^{0}}=1192.83 \pm 0.3 \mathrm{MeV} \\
& M_{\Sigma^{-}}=1197.70 \pm 0.32 \mathrm{MeV}
\end{aligned}
$$

Our results show that the $\mathrm{SU}_{3}$ prediction of equal spacing of the masses within the s-multiplat, $\quad\left(M_{\Sigma^{-}}-M_{\Sigma^{0}}=M_{\Sigma^{0}}-M_{\Sigma^{+}}\right)$, is only approximately satisfied.

Similar results have been obtained by the groups of the University of Maryland [2] and the University of Columbia [3].

\section{REFERENCES}

1. R o s e nf e $1 \mathrm{~d}$ A. H. et al. UCRL $-8030-$ Part 1, June, 1964.

2. Burnstein R. A. et al. Phys, Rev. Lett. (See this Conference Report).

3. S c h mid t P. et al. Report at Conference on Higher energies, 1964. 